\title{
Rationally designed siRNAs without miRNA-like off-target repression
}

\author{
Heeyoung Seok ${ }^{1}$, Eun-Sook Jang ${ }^{2}$ E Sung Wook Chi ${ }^{1, *}$ \\ ${ }^{1}$ Division of Life Sciences, College of Life Sciences and Biotechnology, Korea University, Seoul 02841, ${ }^{2}$ EncodeGEN Co. Ltd., Seoul 06329, \\ Korea
}

Small interfering RNAs (siRNAs) have been developed to intentionally repress a specific gene expression by directing RNA-induced silencing complex (RISC), mimicking the endogenous gene silencer, microRNAs (miRNAs). Although siRNA is designed to be perfectly complementary to an intended target mRNA, it also suppresses hundreds of off-targets by the way that miRNAs recognize targets. Until now, there is no efficient way to avoid such off-target repression, although the mode of miRNA-like interaction has been proposed. Rationally based on the model called "transitional nucleation" which pre-requires base-pairs from position 2 to the pivot (position 6) with targets, we developed a simple chemical modification which completely eliminates miRNA-like off-target repression $(0 \%)$, achieved by substituting a nucleotide in pivot with abasic spacers (dSpacer or C3 spacer), which potentially destabilize the transitional nucleation. Furthermore, by alleviating steric hindrance in the complex with Argonaute (Ago), abasic pivot substitution also preserves near-perfect on-target activity ( 80-100\%). Abasic pivot substitution offers a general means of harnessing target specificity of siRNAs to experimental and clinical applications where misleading and deleterious phenotypes from off-target repression must be considered. [BMB Reports 2016; 49(3): 135-136]

*Corresponding author. E-mail: chi13@korea.ac.kr

http://dx.doi.org/10.5483/BMBRep.2016.49.3.019

Received 1 February 2016

Keywords: Abasic pivot substitution, Ago, miRNA, Off-target effect, siRNA, Spacer

Abbreviations: 2'-OMe, 2'-O-methyl; Ago, Argonaute; HITS-CLIP, high throughput sequencing of RNA isolated by crosslinking and immunoprecipitation; $\mathrm{IC}_{50}$, half maximal inhibitory concentration; miRNA, microRNA; mRNA, messenger RNA; PCSK9, proprotein convertase subtilisin/kexin type 9; RISC, RNA induced silencing complex; RNAi, RNA interference; siRNA, small interfering RNA; UNA, unlocked nucleic acid

Perspective to: Lee et al. (2015), Abasic pivot substitution harnesses target specificity of RNA interference, Nature Communications, 6:10154, doi: 10.1038/ncomms 10154
miRNA plays important roles in regulating gene expression at the post-transcriptional level by inducing RNAi, impacting various biological phenotypes. One strand, termed the 'guide strand', is selected from mature miRNA ( $\sim 22$ base-pairs duplex), and directs the RISC to bind target mRNAs via loading onto Ago, a core catalytic component of RISC. Depending on miRNA sequence, Ago-miRNA complex in RISC recognizes target mRNAs majorly through at least 6 consecutive base-paired matches to the seed region (position 2-8), and reduces their stability and/or translation. To selectively induce RNAi, siRNA is typically designed to be perfectly complementary to a target mRNA, and synthesized as the duplex which can be incorporated into the Ago complex, triggering cleavage of on-target mRNA. Because of its small size, cost-effectiveness and potent activity with a simple procedure, siRNA has been widely used to silence an intended target gene, to study various genes for loss of function, and in therapeutics to knock down disease-causing genes.

Despite the great success of siRNAs, their applications soon faced "off-target" issues. This was because both siRNAs and miRNAs shared the same effector, Ago, which unable to distinguish between the two. As a dual player, siRNA functions similar to miRNA, suppressing the hundreds of off-target transcripts wherever the seed region can make a match. Thus, the use of siRNA always results in inducing off-target effects through the combinatory repression of on-target with hundreds of off-targets, leading to unwanted phenotypes. These miRNAlike off-target repressions cannot be sufficiently avoided by conventional methods, such as the use of appropriate controls, low siRNA concentrations, multiple siRNAs targeting the same gene individually or as a pool. Alternatively, modifications of siRNA with 2'-OMe (position 2) or UNA (position 7) have been empirically found to attenuate the off-target repression, but the effect is marginal, also sacrificing on-target activity. This may be because such modifications have been applied only to the nucleotide backbone rather than to the bases, which directly participate in target recognition. Until now, there is no efficient way to eliminate miRNA-like off-target repression, perpetrating to develop a method rationally based on the mode of Ago-miRNA target recognition.

Functional miRNA-target interaction has been proposed to require "transitional nucleation", a hypothetical mode mediated by base pairs from position 2 to 6 in miRNA, with targets. 
It was postulated during the analytic process to explain the pattern of transcriptome-wide noncanonical "nucleation bulge" sites identified by Ago HITS-CLIP method, where nucleotide in a bulge (position 5-6) of targets should be competent to pair with a nucleotide in position 6 (called 'pivot') of miRNAs (pivot pairing rule). Further supported by structural studies, human Ago2 protein was shown to intrude miRNA after pivot (position 6-7) with isoleucine (I365) in the alpha-helix, disrupting A-form helix structure (position 2-6) which is susceptible to target hybridization. Thus, in theory, any target site pairing to seed region requires to overcome such a kink by extending the transitional nucleation, proceeding from initial recognition to base-pairing toward $3^{\prime}$ end. This notion indicates that basepairing in pivot indeed plays a decisive role, necessitating to form a functional miRNA-target complex.

To avoid the initiation step of miRNA-like off-target recognition, we rationally impaired pivot nucleotide in siRNA by substituting it with a spacer, which functions like a nucleotide backbone but no base, resulting in disruption of pivot hybridization and destabilization of transitional nucleation (Fig. 1). Validated by different approaches such as luciferase reporter assays and transcriptome-wide analyses (RNA-Seq and Ago HITS-CLIP), we proved that abasic pivot substitution with dSpacer (6pi), which contains neither a base nor $2^{\prime}-\mathrm{OH}$, causes no significant miRNA-like off-target repression $(0 \%)$ in all ranges of siRNA concentration and in whole transcriptome. The results were all consistent from the cases of siRNAs used as control (targeting Renillar luciferase), designed for experiments (targeting MAPK14) and developed for therapeutic purposes (targeting PCSK9) as well as miRNAs (miR-124, miR-708 and miR-67). Specifically, miR-124 containing 6pi loses seedmediated transcriptome-wide target interactions, repression activity and biological function inducing neurite outgrowth, whereas other conventional modifications (such as 2'OMe and UNA) have limited potency.

A critical issue regarding any method that blocks off-targets is how effectively on-target activity can be preserved. We validated that siRNAs with dSpacer pivot substitution (siRNA-6pi) efficiently maintain their on-target activity $(\sim 80-100 \%)$ without altering the slicing activity of Ago2. Although there is some variation in its efficiency among different siRNAs ( $\leq$ 9-fold decrease, estimated by $I C_{50}$ ), every siRNA-6pi showed the same on-target activity (100\%) as unmodified versions, in the concentration range generally used for siRNA transfection into cell cultures (10-75 nM). The conservation of on-target activity could be explained by structural observation that dSpacer pivot in Ago-siRNA complex has less potential to cause steric hindrance in the kink after pivot (4.9 $\AA$ from I365, Fig. 1A). By providing adequate space, siRNA-6pi could be beneficial for the stepwise conformational transitions: moving out the kink and widening the central cleft, enabling to be compensated by near-perfect matches to on-target sequences, although its transitional nucleation is destabilized by abasic pivot. As a support, superior conservation of on-target activity was elicited by abasic pivot substitution using C3 spacer, which has the least bulky structure (6.4 $\AA$ from I365). Other bulkier abasic conformations, which either involve rSpacer or cause an abasic bulge, showed less efficient on-target activity.

Until now, miRNA-like off-target repression is considered as inevitable, turning out as huge handicap for clinical applications of siRNA. In lieu of this, we identified unexpected and potentially deleterious off-target phenotypes caused by therapeutic siRNAs targeting PCSK9 (in detail, we found cell cycle arrest in human liver cells and defect in copper metabolism, inducing cell death in mouse liver tissue), which are currently in the development stage for lowering plasma cholesterol. However, such adverse effects were eliminated by applying abasic pivot substitution, while maintaining on-target activity (plasma cholesterol was efficiently reduced as much as the unmodified in mouse studies). By providing a simple solution (abasic pivot substitution) as a rational design, we can now modulate siRNA to be optimally balanced between maintaining on-target activity and losing miRNA-like off-target repression. To harness target specificity of RNAi, abasic pivot substitution should become an essential component of all siRNA experiments and their therapeutic applications.

\section{ACKNOWLEDGEMENTS}

This work was supported by grants from the Korean Health Technology R\&D Project, Ministry of Health and Welfare, Republic of Korea (HI15C3137) to S.W.C and National Research Foundation of Korea funded by the Ministry of Education (NRF-2014R1A1A2A16055016) to H.S.
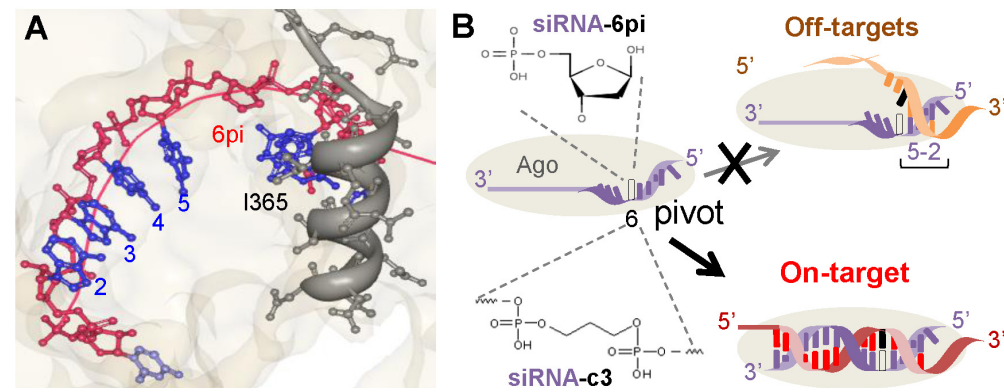

Fig. 1. Abasic pivot substitution in siRNA. (A) Structural model of Ago-siRNA with dSpacer pivot substitution (6pi). (B) A model of pivot substitution with dSpacer or C3 spacer in siRNA, maintaining ontarget interaction but losing miRNA-like off-target recognition. 